\title{
Measuring aesthetic results after facial skin cancer surgery by means of the FACE-Q
}

\author{
SB Kant ${ }^{1}$ (D) $\cdot$ K Mosterd $^{2} \cdot$ NWJ Kelleners-Smeets ${ }^{2} \cdot$ RRWJ Van der Hulst $^{1} \cdot$ A Piatkowski $^{1}$
}

Received: 20 December 2019 / Accepted: 13 January 2020 / Published online: 29 January 2020

(C) The Author(s) 2020

\begin{abstract}
Background Skin cancer is the most commonly occurring type of cancer. However, the influence of facial skin cancer surgery on patients' perceived aesthetic appearance is poorly understood. The objective of this study was to provide an insight into how patients perceive the aesthetic outcome of facial skin cancer surgery by means of a specialised patient-reported outcome measure designed for the aesthetic evaluation of the face: the FACE-Q.

Methods A total of 47 patients with non-melanoma skin cancer who were scheduled for Mohs's micrographic surgery (MMS) or standard surgical excision (SE) were included. These patients filled out three different FACE-Q questionnaires: satisfaction with facial appearance, social function, and satisfaction with outcome. Follow-up was conducted after baseline at 1 month and 3 months post-surgery.

Results No significant differences were detected between baseline and follow-up regarding social function and satisfaction with facial appearance. However, after 3 months, patients were significantly more satisfied with the result of surgery when compared with the 1-month post-surgery follow-up.

Conclusions The perceived aesthetic appreciation of patients does not seem to be significantly influenced by facial skin cancer SE or MMS surgery in this 3-month follow-up study.

Level of evidence: Not ratable.
\end{abstract}

Keywords Facial skin cancer $\cdot$ Facial surgery $\cdot$ Reconstructive surgery $\cdot$ FACE-Q

\section{Introduction}

Skin cancer is the most commonly occurring type of cancer, of which basal cell carcinoma (BCC) is the most common form with an estimated incidence of more than four million cases in the US alone [1]. In the Netherlands, the incidence of BCC is of almost thirty thousand cases each year [2]. The incidence of $\mathrm{BCC}$ is rising annually [3].

Electronic supplementary material The online version of this article (https://doi.org/10.1007/s00238-020-01625-z) contains supplementary material, which is available to authorized users.

SB Kant

sanderkant1@gmail.com

1 Department of Plastic, Reconstructive and Hand Surgery, Maastricht University Medical Center, P Debyelaan 25, 6229HX Maastricht, The Netherlands

2 Department of Dermatology, Maastricht University Medical Center, P Debyelaan 25, 6229HX Maastricht, The Netherlands
Surgery is usually the first choice of treatment for nonmelanoma skin cancer (NMSC). However, it can result in extensive facial defects with noticeable scars, negatively influencing the overall well-being of patients [4].

Surgical treatment techniques for NMSC include standard surgical excision (SE) and Mohs' micrographic surgery (MMS). MMS has been developed to spare the healthy tissue by delivering a comprehensive assessment of resection margins [5].

During the past decade, patient-reported outcome measures (PROMs) are becoming increasingly appreciated when evaluating different therapies in dermatology $[6,7]$.

To determine the health-related quality of life (QOL) after NMSC by means of PROMs, a number of studies have been conducted evaluating these types of tools. In patients with NMSC, promising PROMs with the most potential for clinical application include the Skin Cancer Index, Skindex, Skin Cancer Quality of Life Impact Tool, Skin Cancer Quality of Life questionnaire, and the Facial Skin Cancer Index [8-11]. These tools have proven to 
have a role in the assessment of the overall quality of life of patients with (facial) skin cancer. However, when skin cancer occurs in such a delicate area as the face, it can be insightful to be able to comprehend how the facial appearance perceived by patients is influenced by the surgical removal of skin cancer. For this purpose, the abovementioned QOL tools are not useful as they do not focus on the assessment of facial appearance by patients in detail after the removal of facial skin cancer.

In order to assess (facial) scars, some specific tools have been applied. The Vancouver Scar Scale (VSS), developed to provide a more objective measurement of burn scars, has proven to be a reliable tool and has been widely used for the assessment of scars [12]. However, one of the major disadvantages of this tool is that it does not take patient scar perception into consideration; therefore, it does not evaluate the quality of life of patients. Since 2004, the Patient and Observer Scar Assessment Scale (POSAS) has been developed as a useful alternative tool for assessing scars $[13,14]$. The distinctive characteristic of this tool is that it includes about the opinion of patients regarding their scar. Nevertheless, this tool does not cover the influence that scars have on patients' quality of life [15-17]. Regarding scars after NMSC surgery, a few studies have shown acceptable results in patients' perception $[18,19]$. However, these data about the aesthetic evaluation after NMSC surgery are primarily limited because no validated PROMs were employed in these studies.

Extensive and qualitative data with respect to the patients' perceived facial appearance after face reconstruction after NMSC is lacking in times where there is an increasing emphasis in conserving a decent facial appearance after surgery.

The FACE-Q, developed by Klassen et al. in 2010, is a validated PROM designed to evaluate patients' experience and outcomes after facial aesthetic procedures. In contrast to POSAS, for example, FACE-Q entirely consists of questionnaires that are exclusively filled out by patients. The FACE-Q is composed of more than 40 independently functioning scales in four distinct domains: satisfaction with facial appearance, health-related quality of life, adverse effects, and process of care. It has proven to be a useful tool for evaluating multiple units of the face with respect to aesthetics [20-22]. Therefore, we consider this to be a helpful instrument in assessing patient satisfaction with facial appearance after the removal of facial skin cancer.

\section{Aim}

The aim of this study was to provide more insight in the perceived aesthetic outcome of patients after facial skin cancer surgery. The judgement of aesthetic outcome will take place by means of a specialised aesthetic PRO instrument, the FACE-Q.

\section{Materials and methods}

\section{Study design}

This prospective study was conducted at the Maastricht University Medical Center (MUMC+) between October 2017 and April 2018. Patients undergoing a surgical treatment for a histologically proven facial skin cancer were approached and informed about the study via mail at least 1 week before the surgery was arranged.

\section{Data collection}

For the assessment of their facial image, patients were asked to complete three different FACE-Q questionnaires in total, at baseline and at the two follow-ups. Patients who had no cognitive impairment and who were fluent in Dutch were included. The questionnaires were sent to the patients via mail.

\section{Study population and follow-up}

Patients with different types of skin cancer, including basal and squamous cell carcinomas, intra-epidermal melanomas, and basosquamous carcinomas, were asked to participate. Only patients with malignancies located on the face were included. All patients were treated by a board certified or resident dermatologist by means of surgical excision or MMS. Reconstruction was performed either by the same dermatologist or by a board certified or resident plastic surgeon. Patients who already underwent facial surgery for a different lesion in a period shorter than 6 months prior to the start of this study were excluded. Additionally, patients requiring surgery for multiple skin cancer lesions simultaneously were excluded. Only one procedure per patient was performed. Evaluation took place at the baseline ( 1 to 2 weeks prior to surgery), 1 month, and 3 months post-surgery.

\section{Assessment}

For the assessment of patients' satisfaction with their facial appearance in general and in social situations, FACE-Q questionnaires were used (Appendices 1-3). This tool was chosen because FACE-Q is one of the few tools that meets the current recommendation for the development and validations of 
PROMs [23]. Although the FACE-Q consists of over 40 scales in four domains, it is designed in a way that a subset of FACE-Q scales can be used, as each scale is scored independently. Therefore, it is not necessary to complete all scales.

Scales that were utilised in this study were satisfaction with facial appearance (for the assessment of patients' facial appearance in general), social function (to assess facial appearance in social situations), and satisfaction with result. The three scales were administered at baseline (except for the satisfaction with result questionnaire), 1 month, and 3 months follow-up. These questionnaires are part of the satisfaction with the facial appearance domain, the health-related quality of life domain, and the process of care domain of the FACE-Q, respectively.

Collected baseline characteristics were age, sex, the location of the skin cancer, and the maximum diameter of the skin cancer lesion in millimetres.

\section{Data analysis}

Responses to the FACE-Q questions were scored based on a Likert scale ranging from 1 to 4 . FACE-Q questionnaires contain a table that transforms the sum of each FACE-Q questionnaire to a standardised Rasch transformed score from 0 to 100 . A higher Rasch score indicates a greater satisfaction or quality of life. Mean overall transformed Rasch scores were compared using paired $t$ tests when data was normally distributed and by means of Wilcoxon signed rank test when data was not normally distributed. Subgroup analyses were performed based on the location on the face and size of the defect. Power analysis indicated that a sample size of at least 35 patients was necessary to detect a one-point difference in answers (according to the Likert scale) for FACE-Q questionnaires between the different follow-up moments. A one-point difference includes a change from very or somewhat (dis)satisfied to somewhat or very (dis)satisfied or from somewhat or definitely (dis)agree to definitely or somewhat (dis)agree (see Appendices 1-3). The program SPSS (Statistical Package for Socal Sciences, version 22.0.0) was used for statistical analysis.

\section{Results}

A total of 138 patients were approached, of whom 47 (23 men and 24 women) participated in the study for an overall response rate of $34 \%$. From the initial 47 included patients, 35 filled out the 1-month questionnaires and 42 filled out the final 3 -month questionnaires. The main reason for loss to follow-up is that patients who were lost to follow-up returned the 1- month and 3-month questionnaires too late. A delay of no more than 1 week was considered acceptable by the researchers. Patients who were scheduled for surgery were recruited via mail. Patients who did not return the consent forms were not contacted again for non-participation.

The mean age of the patients was 71 years (ranging from 43 to 85). Forty patients had a basal cell carcinoma, 4 suffered from a squamous cell carcinoma, 2 were diagnosed with intra-epidermal melanoma, and 1 patient had a basosquamous carcinoma. Skin cancer was located on the nose (30\%), eyelids (17\%), frontal or temporal (32\%), and the nasolabial folds (21\%).

Lesion diameters were between 2 and $17 \mathrm{~mm}$, with a mean diameter of $7.9 \mathrm{~mm}$. Surgery was performed on 41 occasions by a (resident) dermatologist and six times by a (resident) plastic surgeon. In 27 cases, Mohs' surgery was used. The rest of the patients were treated by means of surgical excision. In 44 cases, the wounds were primarily closed. In two cases, fullthickness skin grafts were used and one time a Hughes flap was used for the closure of a defect at the lower eyelid. All patient characteristics are presented in Table 1.

Table 1 Patient characteristics

\begin{tabular}{ll}
\hline Mean age (years) & 71 \\
Min & 43 \\
Max & 85 \\
Sex & \\
Male & 23 \\
Female & 24 \\
Location & \\
Frontal/temporal & 15 \\
Nose & 14 \\
Eyelids & 8 \\
Nasolabial & 10 \\
Type of skin cancer & \\
BCC* & 40 \\
SCC* & 4 \\
Intra-epidermal melanoma & 2 \\
Basosquamous carcinoma & 1 \\
Lesion diameter & \\
Min (mm) & 2 \\
Max (mm) & 17 \\
Mean (mm) & 7.9 \\
$<5$ mm & 11 \\
$5-10$ mm & 14 \\
$>10$ mm & 18 \\
\hline
\end{tabular}

*BCC, basal cell carcinoma; $S C C$, squamous cell carcinoma 
Fig. 1 FACE-Q scores at the start of the study ( 1 to 2 weeks prior to surgery) and at the two follow-ups

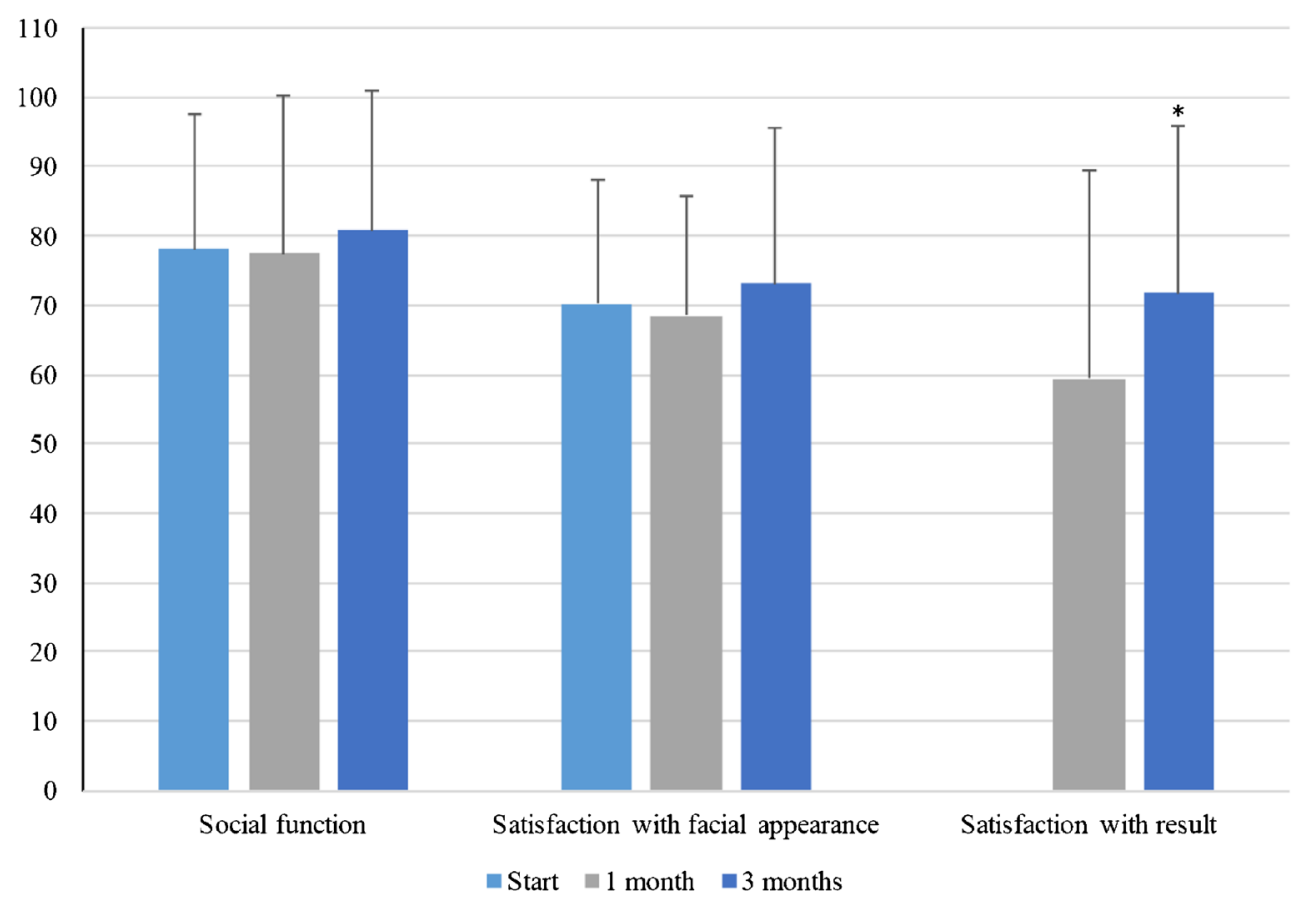

\section{Social function}

Mean transformed Rasch scores regarding patients' satisfaction with their facial appearance in social situations are shown in Fig. 1. No significant differences were found in social function between baseline (78.02, SD:19.41), 1 month (77.42, SD:22.79), and 3 months (80.78, SD:20.25) after surgery.

\section{Satisfaction with facial appearance}

When patients' satisfaction with their facial appearance in general was assessed, again no statistically significant differences were identified between the start of the study and the two follow-up moments (Fig. 1). Rasch scores were 70.27 (SD 17.98) at baseline, 68.53 (SD 17.34) 1 month post-surgery, and 73.05 (SD 22.32) at 3 months post-surgery.

\section{Result satisfaction}

Statistically significant differences in the mean Rasch scores were noticed when patients' satisfaction with the result of surgery was observed at the 1-month and 3-month follow-up. Mean Rasch scores rose from 59.48 (SD 30.05) at 1 month post-surgery to 71.63 (SD 24.11) at 3 months post-surgery, displayed by a $P$ value of 0.041 . When standard surgical excision $(n=20)$ was compared to Mohs' surgery $(n=27)$, no statistically significant differences in satisfaction with the end result were observed (Table 2).

Figure 2 displays the satisfaction with the result surgery for the four different facial sites. The largest increase in satisfaction with the result between 1 and 3 months post-surgery was seen in skin cancer surgeries at the level of the nose (from 57.00, SD 39.12 to 84.92, SD 22.63), followed by skin cancer removal at the eyelids (from 58.67, SD 31.47 to 71.71 , SD 22.02), nasolabial folds (from 46.00 , SD 26.50 to 51.67 , SD 26.49), and frontal/temporal (from 71.90, SD 17.50 to 73.15, SD 16.71). However, the improvement in satisfaction with the result for all facial sites was not considered statistically significant between the two followup moments. Also, no statistically significant differences were found between the four groups at the two followups.

Table 2 Result satisfaction: standard surgical excision (SE) vs. Mohs' micrographic surgery (MMS)

\begin{tabular}{llllll}
\hline & SE & SD & MMS & SD & $P$ \\
\hline 1 month & 51.38 & 28.45 & 64.12 & 30.59 & 0.22 \\
3 months & 64.75 & 27.32 & 76.96 & 20.50 & 0.11 \\
\hline
\end{tabular}


Fig. 2 FACE-Q: satisfaction with outcome scores for four different facial sites at two follow-up moments

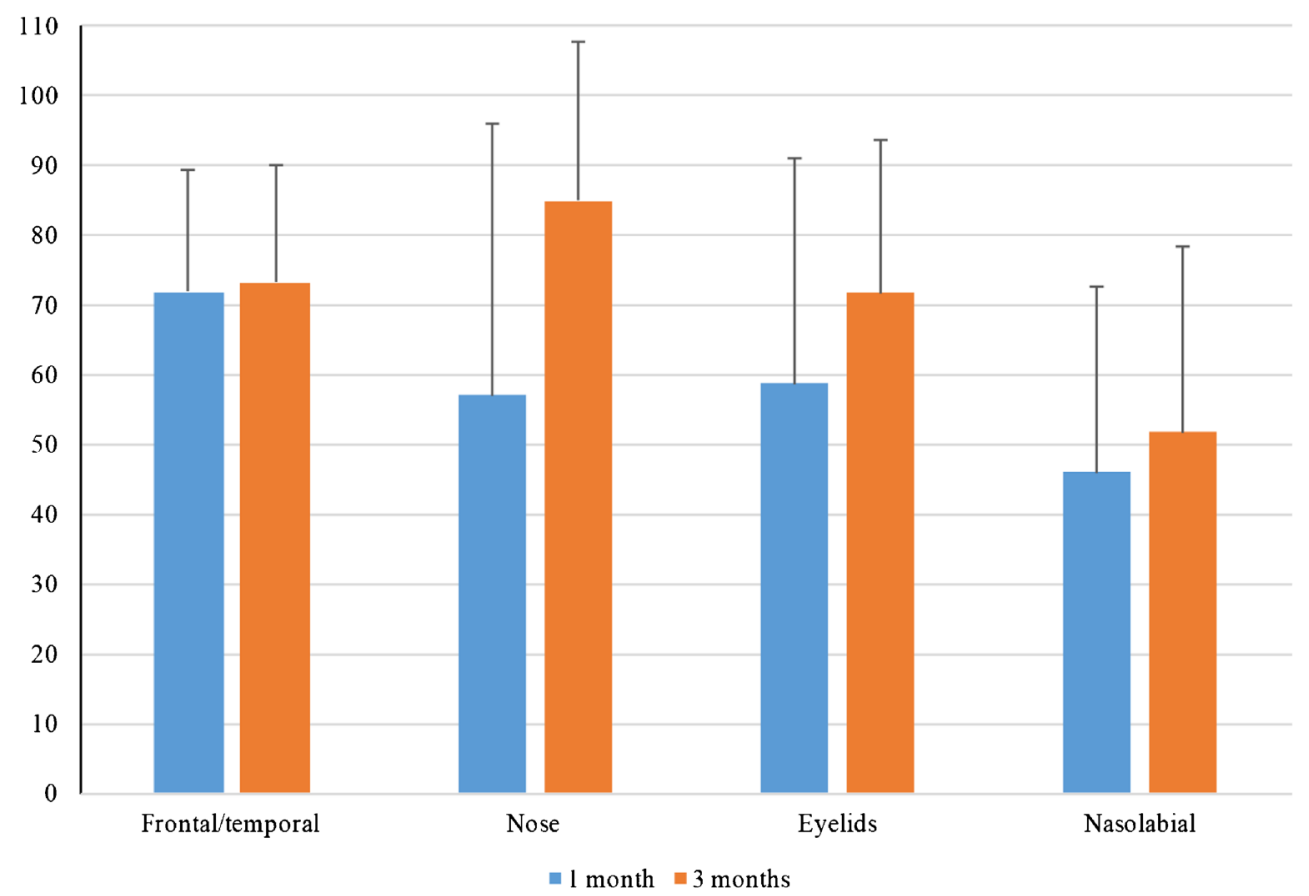

Regarding the size of skin cancer lesions, Fig. 3 shows the satisfaction with the result of surgery. Lesion sizes were divided into smaller than $5 \mathrm{~mm}, 5$ to $10 \mathrm{~mm}$, and larger than $10 \mathrm{~mm}$ lesions. The Rasch scores from patients with lesions larger than $10 \mathrm{~mm}$ displayed a statistically significant increase between 1 month (62.79, SD 21.79) and 3 months (79.60, SD 20.64) post-surgery, displayed by a $P$ value of 0.038 .

Fig. 3 FACE-Q: satisfaction with outcome scores for the size of the skin cancer at two follow-up moments

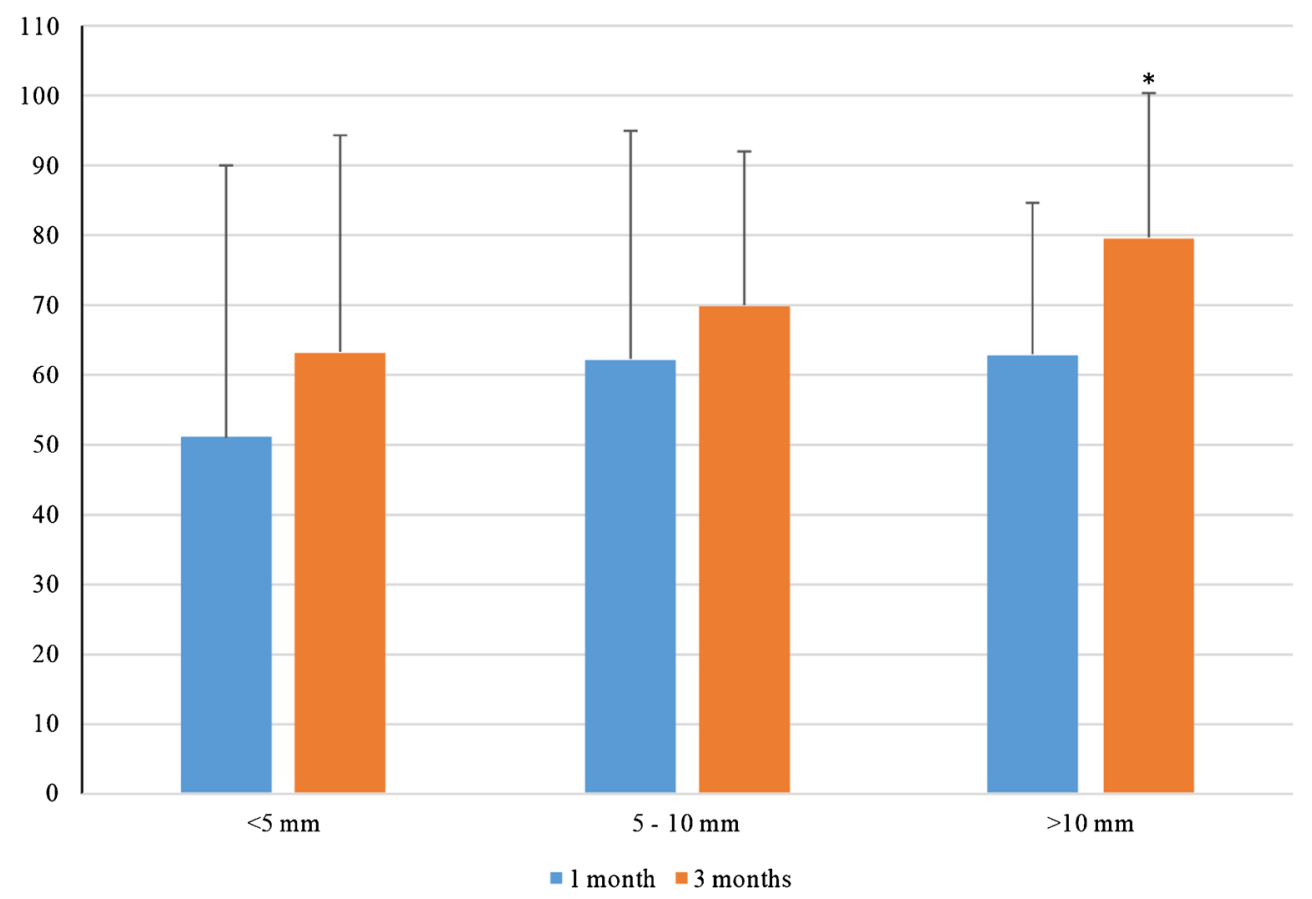


Lesions smaller than $5 \mathrm{~mm}$ (51.00, SD 39.11 at 1 month and 63.18 , SD 31.12 at 3 months) and lesions between 5 and $10 \mathrm{~mm}$ (62.17, SD 32.74 at 1 month and 69.89, SD 22.04 at 3 months) did not show a statistically significant increase in the mean Rasch scores between 1 and 3 months post-surgery ( $P$ values of 0.378 and 0.795 , respectively). Furthermore, no statistically significant differences in result satisfaction were found between all lesion sizes at the two follow-ups.

\section{Discussion}

The major result of this study is that patients are significantly more satisfied with the aesthetic result of facial skin cancer surgery (by means of MMS or SE) at 3 months after the surgery when compared to 1-month post-surgery.

Our results also show that skin cancer surgery does not induce a significant decrease in the patients' perceived overall facial appearance between baseline, 1 month, and 3 months after surgery. These results have been, for the first time, measured by a PROM designed for the aesthetic evaluation of the face: the FACE-Q. FACE-Q has proven to be a helpful and valuable tool in the assessment of facial aesthetic procedures, for instance in the treatment with botulinum toxin, in face lifting procedures, and (non)surgical rhinoplasty [24-26].

The increase in the patients' satisfaction with their facial appearance over the course of 2 months postsurgery could be explained by the hypothesis that in the first 3 months, scars are erythematous and therefore more noticeable, as observed in a study by Bond and colleagues [27]. Furthermore, there is an increase in the net production of collagen until approximately 21 days after the skin injury takes place, giving scars a more thickened appearance. After this, the collagen synthesis rate decreases. Subsequently, capillary redness density decreases as the regression of the inflammatory process occurs and scars pass into the maturation phase, becoming less prominent [28].

The results also show that patients' satisfaction with the outcome of surgery significantly improves in larger defects on the face $(>10 \mathrm{~mm})$ between 1 and 3 months post-surgery. The effect on patients' satisfaction with the surgery outcome over the course of 2 months for smaller defects $(<10 \mathrm{~mm})$ appears to be less noticeable in this study, as no significant differences in patients' satisfaction with the outcome were observed between the two follow-up moments. We hypothesise that patients' expectations about the outcome of the surgery could be influenced by the information about the expected aesthetic result that was expressed by the physician before surgery. Because the selection of patients took place by different physicians, observer bias could have occurred. For a better understanding of the correlation between the size of facial skin cancer and patients' satisfaction with the outcome of the surgery, larger study populations with unambiguous instructions from doctors in future studies are needed.

The study also revealed that no significant differences in patients' satisfaction with the result of therapy were observed between MMS and SE at the two follow-ups. However, this study was not designed to compare SE and MMS with respect to the aesthetic outcome. Considering that MMS does not belong to the standard treatment modalities in most hospitals in Europe yet, the results of this study can also be applicable in practices were the only therapeutic option is SE.

No significant differences were seen with respect to patients' outcome satisfaction when comparing different facial zones. However, in order to assess facial zones or better, the aesthetic subunits of the face where skin cancer surgery has the highest impact on the perceived aesthetic outcome, larger study populations are needed. Also, despite most patients' appreciation with their facial appearance in general and in social situations returns to baseline after 3 months follow-up, a longer follow-up period is preferable to assess the aesthetic impact of NMSC facial surgery more thoroughly.

\section{Conclusion}

This study showed that facial skin cancer surgery by means of SE or MMS is able to conserve the patients' perceived aesthetic appreciation of their face in a 3month follow-up study assessed by means of FACE-Q scales. It also showed that patients' satisfaction with the aesthetic outcome resulted in a significant improvement between the 1- and 3-months post-surgery timeline. 


\section{Compliance with ethical standards}

Conflict of interest Kant SB, Mosterd K, Kelleners-Smeets NWJ, Van der Hulst RRWJ, and Piatkowski A declare that they have no conflict of interest.

Informed consent All participants give their informed consent in writing prior to inclusion in the study.

Ethical approval All procedures performed in studies involving human participants were in accordance with the ethical standards of the institutional research committee of the Maastricht University Medical Center (MUMC+) and with the 1964 Helsinki declaration and its later amendments or comparable ethical standards.

Open Access This article is licensed under a Creative Commons Attribution 4.0 International License, which permits use, sharing, adaptation, distribution and reproduction in any medium or format, as long as you give appropriate credit to the original author(s) and the source, provide a link to the Creative Commons licence, and indicate if changes were made. The images or other third party material in this article are included in the article's Creative Commons licence, unless indicated otherwise in a credit line to the material. If material is not included in the article's Creative Commons licence and your intended use is not permitted by statutory regulation or exceeds the permitted use, you will need to obtain permission directly from the copyright holder. To view a copy of this licence, visit http://creativecommons.org/licenses/by/4.0/

\section{References}

1. Rogers HW, Weinstock MA, Feldman SR, Coldiron BM (2015) Incidence estimate of nonmelanoma skin cancer (keratinocyte carcinomas) in the U.S. population, 2012. JAMA Dermatol 151:10811086

2. Flohil SC, de Vries E, Neumann HA, Coebergh JW, Nijsten T (2011) Incidence, prevalence and future trends of primary basal cell carcinoma in the Netherlands. Acta Derm Venereol 91:24-30

3. Mohan SV, Chang AL (2014) Advanced basal cell carcinoma: epidemiology and therapeutic innovations. Curr Dermatol Rep 3:4045

4. Lee EH (2016) Patient expectations and performance measures in dermatologic surgery. Clin Dermatol 34:111-113

5. Cumberland L, Dana A, Liegeois N (2009) Mohs micrographic surgery for the management of nonmelanoma skin cancers. Facial Plast Surg Clin North Am 17:325-335

6. Rhee JS, Loberiza FR, Matthews BA, Neuburg M, Smith TL, Burzynski M (2003) Quality of life assessment in nonmelanoma cervicofacial skin cancer. Laryngoscope. 113:215-220

7. Gaulin C, Sebaratnam DF, Fernandez-Penas P (2015) Quality of life in non-melanoma skin cancer. Australas J Dermatol 56:70-76

8. Gibbons E, Casanas i Comabella C, Fitzpatrick R (2013) A structured review of patient-reported outcome measures for patients with skin cancer, 2013. Br J Dermatol 168:1176-1186
9. Lee EH, Klassen AF, Nehal KS, Cano SJ, Waters J, Pusic AL (2013) A systematic review of patient-reported outcome instruments of nonmelanoma skin cancer in the dermatologic population. J Am Acad Dermatol 69:e59-e67

10. Burdon-Jones D, Gibbons K (2013) The skin cancer quality of life impact tool (SCQOLIT): a validated health-related quality of life questionnaire for non-metastatic skin cancers. J Eur Acad Dermatol Venereol 27:1109-1113

11. Vinding GR, Christensen KB, Esmann S, Olesen AB, Jemec GB (2013) Quality of life in non-melanoma skin cancer-the skin cancer quality of life (SCQoL) questionnaire. Dermatol Surg 39:1784 1793

12. Sullivan T, Smith J, Kermode J, McIver E, Courtemanche DJ (1990) Rating the burn scar. J Burn Care Rehabil 11: 256-260

13. Mosterd K, Arits AH, Nelemans PJ, Kelleners-Smeets NW (2013) Aesthetic evaluation after non-invasive treatment for superficial basal cell carcinoma. J Eur Acad Dermatol Venereol 27:647-650

14. Liu X, Nelemans PJ, Van Winden M, Kelleners-Smeets NW, Mosterd K (2017) Reliability of the patient and observer scar assessment scale and a 4-point scale in evaluating linear facial surgical scars. J Eur Acad Dermatol Venereol 31:341-346

15. Fearmonti RM, Bond JE, Erdmann D, Levin LS, Pizzo SV, Levinson $H$ (2011) The modified patient and observer scar assessment scale: a novel approach to defining pathologic and nonpathologic scarring. Plast Reconstr Surg 127:242-247

16. Roques C, Teot L (2007) A critical analysis of measurements used to assess and manage scars. Int J Low Extrem Wounds 6:249-253

17. Stavrou D, Haik J, Weissman O, Goldan O, Tessone A, Winkler E (2009) Patient and observer scar assessment scale: how good is it? J Wound Care 18:171-176

18. Petit JY, Avril MF, Margulis A, Chassagne D, Gerbaulet A, Duvillard P, Auperin A, Rietjens M (2000) Evaluation of cosmetic results of a randomized trial comparing surgery and radiotherapy in the treatment of basal cell carcinoma of the face. Plast Reconstr Surg 105:2544-2551

19. Dixon AJ, Dixon MP, Dixon JB (2007) Prospective study of longterm patient perceptions of their skin cancer surgery. J Am Acad Dermatol 57:445-453

20. Klassen AF, Cano SJ, Schwitzer JA, Scott AM, Pusic AL (2015) FACE-Q scales for health-related quality of life, early life impact, satisfaction with outcomes, and decision to have treatment: development and validation. Plast Reconstr Surg 135:375-386

21. Klassen AF, Cano SJ, Scott A, Snell L, Pusic AL (2010) Measuring patient-reported outcomes in facial aesthetic patients: development of the FACE-Q. Facial Plast Surg 26:303-309

22. Klassen AF, Cano SJ, Pusic AL (2016) Use of FACE-Q to measure quality of life following aesthetic facial treatments. JAMA Facial Plast Surg 18:148-149

23. Morley D JC, Fitzpatrick R (2012) A structured review of patientreported outcome measures used in cosmetic surgical procedures: report to department of health. Health Services Research Unit, Department of Public Health, University of Oxford

24. Pascali M, Botti C, Botti G (2020) Face lifting in bald male patients: new trends and specific needs. Plast Reconstr Surg 145:60-69 
25. East C, Badia L, Marsh D, Pusic A, Klassen AF (2017) Measuring patient-reported outcomes in rhinoplasty using the FACE-Q: a single site study. Facial Plast Surg 33:461-469

26. Alouf E, Murphy T, Alouf G (2019) Botulinum toxin type a: evaluation of onset and satisfaction. Plast Surg Nurs 39:148-156

27. Bond JS, Duncan JA, Sattar A, Boanas A, Mason T, O'Kane S, Ferguson MW (2008) Maturation of the human scar: an observational study. Plast Reconstr Surg 121:1650-1658
28. Baum CL, Arpey CJ (2005) Normal cutaneous wound healing: clinical correlation with cellular and molecular events. Dermatol Surg 31:674-686 discussion 86

Publisher's note Springer Nature remains neutral with regard to jurisdictional claims in published maps and institutional affiliations. 